\title{
Generation of DNA nanocircles containing mismatched bases
}

Yu Xiao ${ }^{1,4}$, Caroline Jung ${ }^{1}$, Andreas D. Marx ${ }^{1}$, Ines Winkler ${ }^{1}$, Claire Wyman²,3 ${ }^{2}$ Joyce H.G. Lebbink ${ }^{2,3}$, Peter Friedhoff ${ }^{1}$, and Michele Cristovao ${ }^{2}$

${ }^{1}$ Institute of Biochemistry, Justus-Liebig University, Giessen, Germany, ${ }^{2}$ Department of Cell Biology and Genetics, and ${ }^{3}$ Department of Radiation Oncology, Erasmus Medical Center, Rotterdam, The Netherlands, ${ }^{4}$ Institute of Anatomy and Cell Biology II, Justus-Liebeg University, Aulweg 123, 35392 Giessen, Germany

BioTechniques 259-265 (October 2011) doi 10.2144/000113749

Keywords: DNA mismatch repair; nanocircles; strand discrimination

Supplementary material for this article is available at www.BioTechniques.com/article/113749

The DNA mismatch repair (MMR) system recognizes and repairs errors that escaped the proofreading function of DNA polymerases. To study molecular details of the MMR mechanism, in vitro biochemical assays require specific DNA substrates carrying mismatches and strand discrimination signals. Current approaches used to generate MMR substrates are time-consuming and/or not very flexible with respect to sequence context. Here we report an approach to generate small circular DNA containing a mismatch (nanocircles). Our method is based on the nicking of PCR products resulting in single-stranded 3' overhangs, which form DNA circles after annealing and ligation. Depending on the DNA template, one can generate mismatched circles containing a single hemimethylated GATC site (for use with the bacterial system) and/or nicking sites to generate DNA circles nicked in the top or bottom strand (for assays with the bacterial or eukaryotic MMR system). The size of the circles varied (323 to $1100 \mathrm{bp}$ ), their sequence was determined by the template DNA, and purification of the circles was achieved by ExoI/ExoIII digestion and/or gel extraction. The quality of the nanocircles was assessed by scanning-force microscopy and their suitability for in vitro repair initiation was examined using recombinant Escherichia coli MMR proteins.

The Escherichia coli DNA mismatch repair (MMR) system is responsible for recognizing and repairing base-base mismatches and small slippage errors that escape the proofreading function of the DNA replicative polymerases (1). In this system, MutS recognizes mismatches, recruits MutL, and this complex activates MutH, which nicks the DNA. Next, additional MMR components unwind and remove the incorrect strand and resynthesize the DNA. Up until the point of strand discrimination, MMR is highly conserved from bacteria to humans. The main difference resides in the strand discrimination signal used, as some bacterial systems are MutH-dependent and guided by the methylation status of the DNA at GATC-sequences (2), while most bacterial and the eukaryotic systems do not require MutH-homologs, but rely on preexisting nicks that direct repair (3-5). Despite numerous studies, the MMR system still possesses mechanistic challenges, such as the process by which MMR proteins facil- itate the communication between two physically distant DNA sites (the mismatch and the strand discrimination signal). To answer these questions, mismatched DNA molecules are required. Methods producing linear mismatch-containing DNA substrates have been reported previously (6). However, linear DNA possesses open ends, and proteins that can slide along the DNA, such as MutS (7), can dissociate from the ends of the DNA, rendering the study of the sliding state of proteins and the directionality of the system difficult. Furthermore, these DNA ends may act as entry points for $\mathrm{UvrD}$ helicase resulting in mismatch-provoked MutSL-dependent unwinding and exonuclease digestion of un-nicked linear DNA $(2,8)$. In addition, it is more convenient to follow the DNA nicking by conversion of closed circular (cc) to open circular (oc) DNA rather than using denaturing gels, which are required when analyzing nicking of linear DNA. The use of closed circular DNA (ccDNA) bypasses these difficulties, and several approaches have been published to generate such substrates; however they require the production of phage DNA $(9,10)$, which is time-consuming, or rely on the use of plasmids with the requirement of specific restriction sites for nicking enzymes $(11,12)$. In addition, the published methods use large plasmids containing multiple GATC or nicking sites, which complicate the analysis of distance dependence. Here, we report the development of small circular DNA molecules (nanocircles), into which mismatched bases can be introduced. Strand discrimination signals can be added at specific locations by choosing appropriate sequences to generate hemimethylated GATC-sequences by DNA methyltransferases or a single nick by nicking endonucleases. We show that the current method is generic, simple, and fast. The freedom to choose the DNA template and the size of the nanocircles allows control over the sequence present in the final product. These 
nanocircles are suitable for scanning-force microscopy (SFM), as they are small enough to fall open on the mica, but large enough to clearly recognize protein-DNA complexes and structural changes induced by the proteins. The substrates are shown to be useful for the analysis of MutH nicking activity.

\section{Materials and methods Oligonucleotides}

We purchased the oligonucleotides from Biomers (Ulm, Germany). The sequence of primer P1 was 5'-CTCAAGCTTCACTGCTCATCCTCGGCACCGTCAC-3' (underlined nucleotides are the sequence complementary to the $3^{\prime}$ overhang used in circle formation; bold nucleotides are the recognition sequence for $\mathrm{Nb}$.BtsI) (Figure 1B). The sequence of primer P2 was 5'-AAGCTCGAGCACTGCATTTCCCCATCGGTGATGTC-3'. The sequence of primer P3 was 5'-AAGCTTGAGCACTGCATCTTCCCCATCGGTGATGTC-3'. The sequence of primer $\mathrm{P} 4$ was 5 '-CTCGAGCTTCACTGCCTTCCTTTCGGGCTTTGTTAG-3'. The sequence of primer P5 was 5'-CTCAAGCTTCACTGCATCGCAGAAATCAAAGCTAA-3'. The sequence of primer $\mathrm{P} 6$ was 5'-AAGCTCGAGCACTGCTTGCTCCATTAGCCAGAGCA-3'. Primers P1-P4 have priming sites on $\mathrm{pET}-15 \mathrm{~b}$ (Novagen, Madison, WI, USA) and derivatives thereof. Primers P5 and P6 have priming sites on $\lambda$-DNA (positions 40236-40255 and 41127-41146, respectively).

\section{5-bp ccDNA}

Plasmid pET-15b-XhoI (6) was purified from XL1-Blue $E$. coli cells grown overnight in Luria-Bertani (LB) medium, using the Wizard Plus Miniprep DNA purification system from Promega (Madison, WI, USA). The plasmid was used as a template for PCR with the following combination of primers: P1 and P2 for G:T (mismatch) and P1 and P3 for A:T (homoduplex). For the PCR, $0.4 \mu \mathrm{M}$ forward and reverse primers, $0.1 \mathrm{ng}$ pET15b template, $1 \mathrm{mM}$ dNTPs, $1 \times$ Pfu DNA polymerase buffer, and 5 U Pfu DNA Polymerase (Fermentas, Vilnius, Lithuania) were mixed in a final volume of $100 \mu \mathrm{L}$ and subjected to the following PCR cycle conditions: $94^{\circ} \mathrm{C}$ for $5 \mathrm{~min}, 20$ cycles of $94^{\circ} \mathrm{C}$ for $0.5 \mathrm{~min}, 55^{\circ} \mathrm{C}$ for $0.5 \mathrm{~min}, 68^{\circ} \mathrm{C}$ for $1 \mathrm{~min}$, and a final extension at $68^{\circ} \mathrm{C}$ for $7 \mathrm{~min}$. This reaction was prepared 14 times. The PCR products from multiple $100-\mu \mathrm{L}$ reactions were purified over four SV minicolumns from the Wizard SV gel and PCR cleanup



Figure 1. The approach used to generate closed circular DNA containing mismatched bases. (A) Closed circular DNA of four different sizes $(323,505,932$, and 1100 bp) were synthesized by generating a linear DNA with two complementary overhangs (produced by one nicking enzyme, Nb.Btsl) and a base-base mismatch located in the overhang. (B) Detail of the nicking step: Nb.Btsl nicks $5^{\prime}$ to CACTGC sequence creating two 9-bp 3' overhangs on each side of the linear PCR product, which can then anneal and form a circle (black bases). The primers used as forward and reverse for the different circles are shown above or below the annealing site. The G:T mismatch that is introduced by primers $\mathrm{P} 1$ and $\mathrm{P} 2$ in the 505-bp circle is boxed. (C) M.Taql methylation site and MutH recognition sequence (in bold). (D) Nicking sites for Nb.Bpu10I and Nt.Bpu10I.

system (referred to as the reaction cleanup kit; Promega).

After synthesis, the PCR product was nicked with $\mathrm{Nb}$.BtsI by mixing approximately $4 \mu \mathrm{g}$ DNA with $0.1 \mathrm{mg} / \mathrm{mL}$ BSA, $20 \mathrm{U}$ Nb.BtsI, $1 \times$ NEB buffer 4 (New England Biolabs, Ipswich, MA, USA) in a final volume of $100 \mu \mathrm{L}$, and incubation overnight at $37^{\circ} \mathrm{C}$, followed by incubation at $65^{\circ} \mathrm{C}$ for $20 \mathrm{~min}$ (Fermentas). This reaction was prepared 11 times. Immediately afterwards, the digested PCR product was purified with three minicolumns from the reaction cleanup kit. After purification, approximately $2.5 \mu \mathrm{g}$ digested PCR products were ligated with $4 \mathrm{U}$ T4 DNA ligase and $1 \times$ T4 DNA ligase buffer in a final volume of $200 \mu \mathrm{L}$ overnight at room temperature (Fermentas). The reaction was prepared 10 times and purified over three minicolumns by the reaction cleanup kit.

To remove nicked and linear by-products from the closed DNA circle, the following reaction was prepared: ligated DNA (approximately $5 \mu \mathrm{g}), 1 \times$ buffer $\mathrm{R}(10 \mathrm{mM}$ Tris- $\mathrm{HCl}$, $10 \mathrm{mM} \mathrm{MgCl} 2,100 \mathrm{mM} \mathrm{KCl}$ ) with BSA $(0.1 \mathrm{mg} / \mathrm{mL}), 1 \times$ reaction buffer for ExoI, $60 \mathrm{U}$ ExoI, and $240 \mathrm{U}$ ExoIII (Fermentas), in a final volume of $60 \mu \mathrm{L}$. The reaction was incubated at $37^{\circ} \mathrm{C}$ for $1 \mathrm{~h}$, followed by heat inactivation $\left(15 \mathrm{~min}\right.$ at $\left.65^{\circ} \mathrm{C}\right)$. The DNA was again purified over minicolumns from the reaction cleanup kit. The product was analyzed on a $2 \%$ agarose gel (run with a 




Figure 2. Agarose gel showing the product obtained after each reaction step during the generation of the 323-bp circle. PCR (lane 1), nicked with Nb.Btsl (lane 2), ligated with T4 ligase (lane 3), and purified by digestion with Exol and Exolll (lane 4), and by gel extraction (lane 5). CC, closed circle; OC, open circle; DNA. The $2 \%$ agarose gel was run in the presence of ethidium bromide. The marker used was pUC8 Marker Mix, manufactured by Fermentas.

Tris-phosphate-EDTA buffer). To separate the nicked circular from the closed circular DNA, the sample was run in the presence of $0.4 \mathrm{mg} / \mathrm{mL}$ ethidium bromide. The desired product was purified from a $2 \%$ agarose gel using the reaction cleanup kit.

\section{3-bp ccDNA}

The smaller circle was obtained by the same procedure as the 505-bp circles, using primers P4 (forward) and P3 (reverse), generating an $\mathrm{A}: \mathrm{C}$ mismatch.

\section{0-bp ccDNA}

The larger circle was obtained by the same procedure as the 505-bp and 323-bp circles, using primers $\mathrm{P} 1$ and $\mathrm{P} 2$, but using as a PCR template the plasmid LNgoCTD (13) purified from XL1-Blue E. coli cells grown overnight in LB medium. The PCR reactants were mixed as described above and subjected to the following PCR cycle conditions: $94^{\circ} \mathrm{C}$ for $5 \mathrm{~min}$, followed by 20 cycles of $94^{\circ} \mathrm{C}$ for $0.5 \mathrm{~min}, 55^{\circ} \mathrm{C}$ for 0.5 $\min , 68^{\circ} \mathrm{C}$ for $2 \mathrm{~min}$, and a final extension at $68^{\circ} \mathrm{C}$ for $7 \mathrm{~min}$. The remaining protocol was left unchanged except for the ExoI and ExoIII incubation step, in which $400 \mathrm{U}$ instead of $240 \mathrm{U}$ ExoIII were used.

\section{2-bp hemimethylated ccDNA}

Togenerate a circular DNA containing a single hemimethylated GATC-site or a nick in either the top or the bottom strand, we used $\lambda$-DNA
$(0.6 \mu \mathrm{g} / 100 \mu \mathrm{L})$ as a template. The target sequence (position 40236-41146) contains TCGATC and ACCTAAGC sequences that allow methylation of the adenines at the TCGA site by Taq DNA methyltransferases or nicking with Nt.Bpu10I or Nb.Bpu10I (Fermentas) (Figure 1, D and E). Primers P5 and $\mathrm{P} 6$ were used for PCR $\left(94^{\circ} \mathrm{C}\right.$ for $\left.5 \mathrm{~min}\right)$ followed by $25 \mathrm{cycles}\left(94^{\circ} \mathrm{C}\right.$ for $0.5 \mathrm{~min}, 53^{\circ} \mathrm{C}$ for $1 \mathrm{~min}, 68^{\circ} \mathrm{C}$ for $2.5 \mathrm{~min}$ ), and finally $68^{\circ} \mathrm{C}$ for $7 \mathrm{~min}$, in which Phusion High Fidelity Polymerase (New England Biolabs) was used instead of $P f u$. The remaining protocol was left unchanged from that used for the 1100-bp circle.

\section{MutH activation assay}

MutS, MutL, and MutH were purified as $\mathrm{N}$-terminal His-tagged proteins using Ni-NTA affinity chromatography and gel filtration, essentially as described (14-16). MutH and MutL were premixed and incubated on ice for $20 \mathrm{~min}$ in $10 \mathrm{mM}$ Tris- $\mathrm{HCl}, \mathrm{pH} 7.5,10 \mathrm{mM} \mathrm{MgCl}_{2}, 150 \mathrm{mM}$ $\mathrm{KCl}, 0.1 \mathrm{mg} / \mathrm{mL} \mathrm{BSA}$, and $1 \mathrm{mM}$ ATP. The reaction was started by the addition of MutS and DNA and incubation at $37^{\circ} \mathrm{C}$. The final concentration of each component was $500 \mathrm{nM}$ MutH, MutL, and MutS, and 20 nM DNA. Aliquots $(10-\mu \mathrm{L})$ were taken at certain time points $(0,0.5,1,2,10,20$, and $60 \mathrm{~min}$ ), and the reaction was stopped by the addition of $10 \mu \mathrm{L}$ loading buffer $(250$ mM EDTA, 25\% sucrose, $1.2 \%$ SDS, $0.1 \%$ bromophenol blue, $0.1 \%$ xylene cyanol FF). The reaction products were separated on a $2 \%$ agarose gel in Tris-phosphate-EDTA buffer in the presence of $0.4 \mathrm{mg} / \mathrm{mL}$ ethidium bromide.

Scanning-force microscopy

DNA preparations were diluted in deposition buffer (10 mM HEPES, pH 7.5, and $10 \mathrm{mM}$ $\mathrm{MgCl}_{2}$ ) and kept at $37^{\circ} \mathrm{C}$. For MutS-DNA complexes, 9 ng 505-bp homoduplex circle, 9 ng 1100-bp heteroduplex circle, and 4 nM MutS were incubated at room temperature for $5 \mathrm{~min}$, in assembly buffer (10
$\mathrm{mM}$ HEPES, pH 7.5, $10 \mathrm{mM} \mathrm{MgCl}_{2}$ and $150 \mathrm{mM} \mathrm{KCl}$ ). The reaction mixture was then diluted 1:7 in $20 \mu \mathrm{L}$ deposition buffer, and the $20 \mu \mathrm{L}$ were deposited onto freshly cleaved mica. After a 1-min incubation, the mica was rinsed with MilliQ water (Millipore, Billerica, MA, USA) and dried with filtered air. Samples were imaged in air at room temperature by tapping mode using a Nanoscope III (Digital Instruments, Santa Barbara, CA, USA). Uncoated silicon pointprobe tips were type $\mathrm{N}$, resonance frequency $200-400 \mathrm{kHz}$, force constant $25-75 \mathrm{~N} / \mathrm{m}$ (ST Instruments, Groot-Ammers, The Netherlands). Images were collected at $1 \times$ $1 \mu \mathrm{m}$ or $2 \times 2 \mu \mathrm{m}$ and processed only by flattening to remove background slope using Nanoscope software.

\section{SFM-based length analysis}

The length of the circles was determined using manual tracing from images imported into Image SXM 1.69 (National Institutes of Health, IMAGE version modified by Steve Barret, Surface Science Research Center, University of Liverpool, Liverpool, UK). For each circle preparation, the average length of at least 100 different circles was used to calculate the length distribution, the length was converted into base pairs (assuming a B-DNA conformation where the distance between base pairs is $3.4 \AA$ ), and a function describing a Gaussian distribution was fit to the data using Origin 7.5.

\section{Results and discussion \\ Generation of mismatch- containing nanocircles}

The approach used to generate mismatchcontaining nanocircles is outlined in Figure 1. A long PCR product is generated (Figure 1A, a) containing one Nb.BtsI nicking site (NN $\downarrow$ CACTGC) at each $5^{\prime}$ end (Figure 1A, b).pET-15b-XhoI was used as a template with the following set of primers: $\mathrm{P} 4$ plus $\mathrm{P} 3$ (A:C-323; Supplementary Figure S1), P1 and

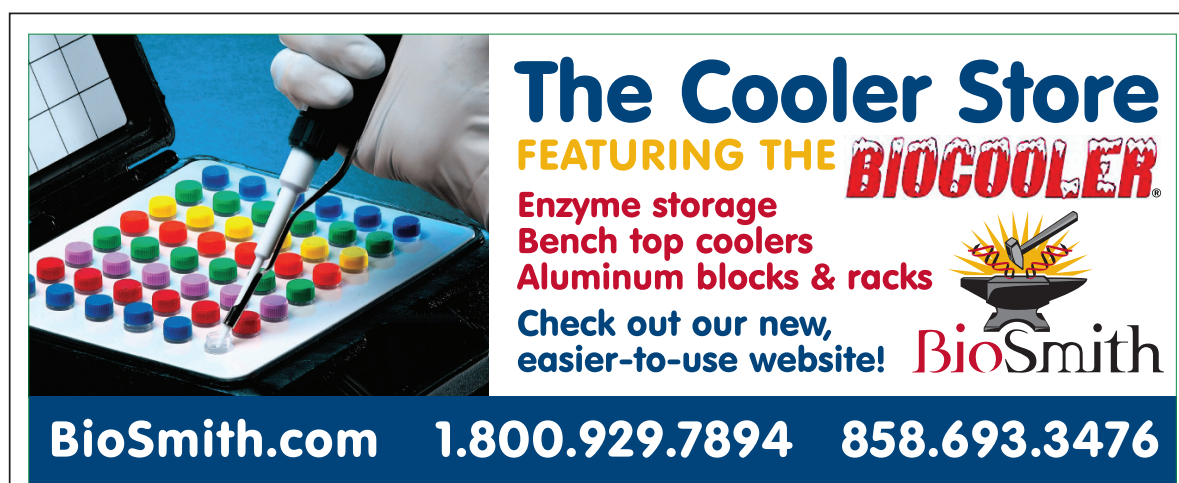


P2 (G:T-505; Supplementary Figure S2) and P1 plus P3 (A:T-505). To generate the linear DNA required for the 1100-bp circle with a G:T mismatch, LNgoCTD was used as a template with primers P1 and P2 (G:T-1100, Supplementary Figure S3). Once the linear product is obtained via PCR, the remaining steps are identical for the four different sized circles. As the PCR primers carry specific nicking sites, the linear DNA was nicked at both ends by $\mathrm{Nb}$.BtsI, generating a linear DNA with two complementary 3 ' overhangs. The overhangs anneal to form a circle, and as they have noncomplementary bases in one position within the overlapping single-strand ends, a mismatch is introduced (Figure 1A, c). Ligation with T4 ligase seals the nick (Figure 1A, d; see Figure 1B for details on overhang annealing). The size of the PCR product dictates the size of the circle. Cyclization of the DNA depends on the $j$ factor, the ring closure probability, which is a ratio of the equilibrium constants for cyclization and for bimolecular association via the cohesive ends; the latter depends on the persistent length $P$ of the DNA (17). Molecules shorter than $P(\sim 150$ bp $)$ will not spontaneously bend to form a circle, and $j$ decreases for molecules smaller than 200 bp and larger than 1000 bp (17). For this reason, we chose to generate circles between 323 and 1100 bp. After ligation, the reaction product was incubated with ExoI, to remove single-stranded DNA, and ExoIII, to remove double-stranded linear and nicked circular DNA. Optionally, circles can be purified by gel extraction or $\mathrm{CsCl}$ gradient centrifugation. Figure 2 shows the 323-bp circle generation, purified by enzymatic digestion and gel extraction. Lanes 1 and 2 show the product after the PCR and nicking reaction, respectively. In lane 3 , in addition to the desired covalently closed DNA, which runs at the same position as the 242-bp marker band, multiple ligation products are observed. These resulted from the intermolecular ligation between the complementary overhangs on different PCR products, leading to the formation of longer products (running higher than $242 \mathrm{bp}$ ). At this point, the enzymatic digestion by ExoI and ExoIII efficiently removes unwanted linear and open circular reaction byproducts (compare lane 3 and lane 4). An additional gel purification step removes the remaining open circle products (lane 5). A small amount of linear DNA is still present, which will be observed in the SFM images (see below). The yield from a typical reaction to generate a nanocircle is about $20 \%$ of the initial amount of PCR product (not shown).

The nanocircle containing the strand discrimination signals (G:T-932, Supplementary Figure S4) was generated using $\lambda$-DNA as a template and primers P5 and


I


$J$
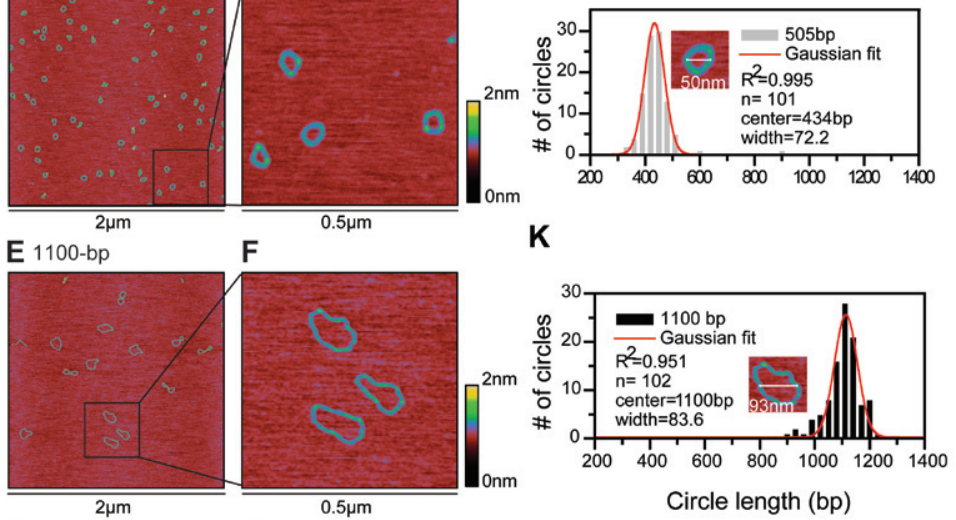

G A:T-505+G:T-1100 H


Circle length (bp)

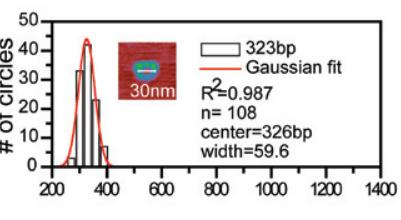



Figure 3. SFM images and length analysis of different sized circles and MutS-DNA complexes. $(A-H)$ Free DNA or MutS-DNA complexes were deposited onto mica and imaged by tapping mode in air. (A) A:C-323 DNA circle at $1 \times 1 \mu \mathrm{m}$ scale. (B) Zoom of the 323-bp circles. (C) G:T-505 DNA circle at $2 \times 2 \mu \mathrm{m}$ scale. (D) Zoom of the 505-bp circles. (E) G:T-1100 DNA circle at $2 \times 2 \mu \mathrm{m}$ scale. (F) Zoom of the 1100-bp circles. (G) Mixture of the A:T-505 and the G:T-1100 circles in the presence of MutS at a $2 \times 2 \mu \mathrm{m}$ scale. $(\mathrm{H})$ Zoom of a complex between MutS and a G:T-1100 substrate. (A-F) The colored bars represent the height from 0 to $2 \mathrm{~nm}$. $(\mathrm{G}-\mathrm{H})$ The color bars represent the height from 0 to $4 \mathrm{~nm}$. (I-K) Length distribution obtained after analysis of the circles imaged with SFM. (I) A:C-323, (J) G:T-505, and (K) G:T-1100 circles. A Gaussian equation was fit to the distributions (red curve). $n$ corresponds to the number of measured circles; center and width are the center and width of the Gaussian distribution.

P6. The primers were chosen so that a single MutH recognition site (GATC) was present overlapping with a M.TaqI methylation site (TCGA). M.TaqI was used to methylate the adenine base at the N6-position to generate a hemimethylated substrate [TCGA(me) TC] (Figure 1C). The presence of a Bpu10I site in the amplicon allows nicking of either the top strand by Nb.Bpu10I or the bottom strand by Nt.Bpu10I (Figure 1D and Supplementary Figure S5). The mismatch disrupted a XhoI and HindIII recognition site (Supplementary Figure S5), and the site that will be restored depends on the base corrected by the mismatch repair system (XhoI in case of G:C and HindIII in case of A:T), as demonstrated elsewhere (18).

\section{Quality assessment}

To verify the size, uniformity, and purity of the DNA nanocircles, we analyzed the circular DNA with SFM (Figure 3). The
DNA preparations were clean and homogeneous, and the majority of the DNA consists of homogeneous circles, with a minority of shorter or longer linear DNA fragments present. The contour length of the circles was measured, and a Gaussian equation was fit to the distribution of circle lengths obtained (Figure 3, K-N). We observed a narrow distribution with an average circle size in good agreement with the theoretical length. The deviation between theoretical and experimental length varies only between $1 \%$ and $14 \%$. To convert the length from nanometers into base pairs, we assumed B-form for the DNA ( $3.4 \AA$ / bp). Simultaneous incubation of MutS with G:T-1100 and A:T-505 circles showed 50\% more binding on the heteroduplex substrate (out of 88 DNA-MutS complexes observed, 29 were on homoduplex, and 59 were on heteroduplex circles). It has been suggested that MutS recognizes mismatches by 
probing the ability of the DNA to bend, due to local defects in base pairing and stacking (19-21). Small circles are intrinsically bent $\left(360^{\circ}\right.$ spread over $505 \mathrm{bp}, 0.7^{\circ}$ per bp). This could lead to small local distortions in base stacking and increased MutS affinity for homoduplex nanocircles, specifically under low salt conditions, where the discrimination between homo- and heteroduplex is lower (the final $\mathrm{KCl}$ concentration after dilution in the deposition buffer is 21 $\mathrm{mM})$ (22).

Finally, to determine the efficiency of our procedure, we recorded several images of the G:T-932 circle and manually counted the different DNA structures present. Eighty-three percent of 487 DNA 932-bp nanocircle (Supplementary Figure S6), showing the efficiency of our method. molecules analyzed correspond to the

Suitability for the mismatch repair system

The heteroduplex circles were tested for in vitro mismatch-dependent $\mathrm{MutH}$ activation (E. coli system). In this assay, MutS binds specifically to a mismatch and recruits MutL in an ATP-dependent manner. The complex of DNA-MutS-MutL activates the sitespecific endonuclease activity of $\mathrm{MutH}$. The generation of nicked products from circular DNA can be followed on an agarose gel in the presence of ethidium bromide. Figure 4, A-C, shows the results of the MutH activation assay on the A:C-323, G:T-505, and G:T-1100 mismatch-containing circles. All three unmethylated circles were cleaved by $\mathrm{MutH}$, and an increase in the reaction rate with increase of circle length was observed. Note the difference between open circle (first nicking event) and linear (second nicking



Figure 4. Analysis of the mismatch-dependent activation of MutH endonuclease activity on the different mismatched circles. (A) The different DNA forms expected. (B) A:C-323, (C) G:T-505, and (C) G:T:1100. Circular DNA (20 nM) was incubated at $37^{\circ} \mathrm{C}$ in the presence of $500 \mathrm{nM}$ MutS, 500 nM MutL, $500 \mathrm{nM}$ MutH, and $1 \mathrm{mM}$ ATP. The reaction was stopped with loading buffer containing SDS after $0,0.5,1,2,10,20$, and 60 min incubation, and the MutH activation was scored by the appearance of the open circle form (OC; first nicking event by MutH) and the linear form ( $\mathrm{L}$; second nicking event by MutH). (E) Comparison of MutHLS reaction with unmethylated and hemimethylated 932-bp substrate. Note that when the substrate was hemimethylated, faster conversion of the closed circle (CC) form to the OC form was observed, but no formation of a linear form. The marker used was pUC8 Marker Mix, manufactured by Fermentas. event) at $10 \mathrm{~min}$ for all three substrates. It is known that recognition and signaling of mismatches by MutS requires kinking and unbending of the DNA at the mismatch (19-21). The slower cleavage rate of the 323-bp circle implies that this conformational change is inhibited in smaller circles in comparison to larger circles. This suggests that a series of circles with different sizes can be useful to study the effect of DNA conformational changes on protein function (23). Using template DNA containing a M.TaqI (TCGA) site overlapping with a GATC site, it was possible to generate hemimethylated DNA. On this mismatched DNA circle, the nicking activity of $\mathrm{MutH}$ is enhanced, resulting from a tighter interaction with hemimethylated GATC sites (24), but limited to the unmethylated bottom strand (Figure 4E and Supplementary Figure S7).

In summary, the method we present is an easy and straightforward approach to generate small mismatched DNA substrates that can be used to analyze the activity of MMR proteins, including relatively uncharacterized members of the MutS-homologs family (such as MSH8, identified in Euglenozoa) (25). As this approach does not require single-stranded phage DNA or specific restriction sites, the substrate sequence and size can be adapted to the particular needs. We show that we can easily obtain circles with different sizes, different mismatches, and either methyl- or nick-directed (top or bottom) strand discrimination signals. In addition, circles with varying sizes between 200 and $1000 \mathrm{bp}$ are easily obtained, providing a quick and easy method to study the influence of constrained DNA structure on protein function (23).

\section{Acknowledgments}

This research was funded by the European Community's Seventh Framework Programme (FP7/2007-2013) under grant agreement no. HEALTH-F4-2008-223545, the German Science Foundation (GRK 1384 and FR1495/4-1 to P.F.), and the Nederlandse Organisatie voor Wetenschappelijk Onderzoek-Chemische Wetenschappen (VIDI 700.58.428 to J.L. and VICI 700.56.441 to C.W.).

\section{Competing interests}

The authors declare no competing interests.

\section{References}

1. Li, G.M. 2008. Mechanisms and functions of DNA mismatch repair. Cell Res. 18:85-98. 


\section{BioTechniques}

2. Au, K.G., K. Welsh, and P. Modrich. 1992. Initiation of methyl-directed mismatch repair. J. Biol. Chem. 267:12142-12148.

3. Su, S.S., R.S. Lahue, K.G. Au, and P. Modrich. 1988. Mispair specificity of methyl-directed DNA mismatch correction in vitro. J. Biol. Chem. 263:68296835.

4. Lu, A.L., S. Clark, and P. Modrich. 1983. Methyl-directed repair of DNA base-pair mismatches in vitro. Proc. Natl. Acad. Sci. USA 80:4639-4643.

5. Holmes, Jr., J., S. Clark, and P. Modrich. 1990. Strand-specific mismatch correction in nuclear extracts of human and Drosophila melanogaster cell lines. Proc. Natl. Acad. Sci. USA 87:5837-5841.

6. Thomas, E., A. Pingoud, and P. Friedhoff. 2002. An efficient method for the preparation of long heteroduplex DNA as substrate for mismatch repair by the Escherichia coli MutHLS system. Biol. Chem. 383:1459-1462.

7. Acharya, S., P.L. Foster, P. Brooks, and R. Fishel. 2003. The coordinated functions of the E. coli MutS and MutL proteins in mismatch repair. Mol. Cell 12:233-246.

8. Runyon, G.T., D.G. Bear, and T.M. Lohman. 1990. Escherichia coli helicase II (UvrD) protein initiates DNA unwinding at nicks and blunt ends. Proc. Natl. Acad. Sci. USA 87:6383-6387.

9. Corrette-Bennett, S.E. and R.S. Lahue. 1999. Mismatch repair assay. Methods Mol. Biol. 113:121-132.

10.Schanz, S., D. Castor, F. Fischer, and J. Jiricny. 2009. Interference of mismatch and base excision repair during the processing of adjacent $\mathrm{U} / \mathrm{G}$ mispairs may play a key role in somatic hypermutation. Proc. Natl. Acad. Sci. USA 106:5593 5598.

11. Wang, H. and J.B. Hays. 2000. Preparation of DNA substrates for in vitro mismatch repair. Mol. Biotechnol. 15:97-104.

12. Drost, M., J.B. Zonneveld, L. van Dijk, H. Morreau, C.M. Tops, H.F. Vasen, J.T. Wijnen, and N. de Wind. 2010. A cell-free assay for the functional analysis of variants of the mismatch repair protein MLH1. Hum. Mutat. 31:247-253.

13. Duppatla, V., C. Bodda, C. Urbanke, P. Friedhoff, and D.N. Rao. 2009. The C-terminal domain is sufficient for endonuclease activity of Neisseria gonorrhoeae MutL. Biochem. J. 423:265-277.

14. Toedt, G.H., R. Krishnan, and P. Friedhoff. 2003. Site-specific protein modification to identify the MutL interface of MutH. Nucleic Acids Res. 31:819-825.

15. Ahrends, R., J. Kosinski, D. Kirsch, L. Manelyte, L. Giron-Monzon, L. Hummerich, O. Schulz, B. Spengler, and P. Friedhoff. 2006. Identifying an interaction site between MutH and the C-terminal domain of MutL by crosslinking, affinity purification, chemical coding and mass spectrometry. Nucleic Acids Res. 34:3169-3180.

16. Manelyte, L., C. Urbanke, L. Giron-Monzon, and P. Friedhoff. 2006. Structural and functional analysis of the MutS C-terminal tetramerization domain. Nucleic Acids Res. 34:5270-5279.

17. Shore, D., J. Langowski, and R.L. Baldwin. 1981. DNA flexibility studied by covalent closure of short fragments into circles. Proc. Natl. Acad. Sci. USA 78:4833-4837.

18. Lahue, R.S., K.G. Au, and P. Modrich. 1989. DNA mismatch correction in a defined system. Science 245:160-164.

19. Lamers, M.H., A. Perrakis, J.H. Enzlin, H.H. Winterwerp, N. de Wind, and T.K.Sixma. 2000. The crystal structure of DNA mismatch repair protein MutS binding to a $\mathrm{G} \times \mathrm{T}$ mismatch. Nature 407:711-717.

20. Obmolova, G., C. Ban, P. Hsieh, and W. Yang. 2000. Crystal structures of mismatch repair protein MutS and its complex with a substrate DNA. Nature 407:703-710.

21. Yang, W. 2008. Structure and mechanism for DNA lesion recognition. Cell Res. 18:184-197.

22. Blackwell, L.J., K.P. Bjornson, D.J. Allen, and P. Modrich. 2001. Distinct MutS DNA-binding modes that are differentially modulated by ATP binding and hydrolysis. J. Biol. Chem. 276:34339-34347.

23. Parvin, J.D., R.J. McCormick, P.A. Sharp, and D.E. Fisher. 1995. Pre-bending of a promoter sequence enhances affinity for the TATA-binding factor. Nature 373:724-727.

24. Lee, J.Y., J. Chang, N. Joseph, R. Ghirlando, D.N. Rao, and W. Yang. 2005. MutH complexed with hemi- and unmethylated DNAs: coupling base recognition and DNA cleavage. Mol. Cell 20:155-166.

25. Sachadyn, P. 2010. Conservation and diversity of MutS proteins. Mutat. Res. 694:20-30.

Received 11 February 2011; accepted 25 August 2011.

Address correspondence to Michele Cristovao, Department of Cell Biology and Genetics, Erasmus Medical Center, Dr. Molewaterplein 50, 3015 GE Rotterdam, The Netherlands.e-mail: m.cristovao@erasmusmc.nl

To purchase reprints of this article, contact: biotechniques@fosterprinting.com

\section{Submit Your Methods}

Do you have a manuscript illuminating a new method, a substantive modification to an existing method, or an innovative application to a new model or scientific question? If so, submit them now to BioTechniques!

BioTechniques offers a rapid peer-review process; time to first decision is often less than 4 weeks. Manuscripts are generally published in print within 3 months of acceptance.

Publishing with BioTechniques means your work reaches the right target audience of more than:

$\mathbf{8 0 , 0 0 0}$ print subscribers

$\mathbf{8 0 , 0 0 0}$ newsletter subscribers

66,000 unique web visitors each month

We are particularly interested in methods from:

- Genomics and Epigenetics

- Molecular Biology

- Proteomics

- Systems Biology

- Stem Cell Biology

- Microscopy

Biotechniques also accepts image submissions of relevant scientific quality and appeal for use on our cover. 